\title{
Load Balancing Through Intermediate Gateway Traffic Sharing Model (IGTSM) for WMN
}

\author{
Neeraj Paliwal \\ Associate Professor \\ Dept. of CSE, OU University \\ Indore (M.P)
}

\begin{abstract}
Ad hoc networks are a temporary network that consists of a set of similar mobile nodes that move freely and independently and communicate with other node via wireless links. The performance of ad hoc wireless network routing protocol is increased when the motion of mobile nodes is reduced while considering the performance factors such as packet delivery ratio and routing overhead. The gateway can be considered as centrally located nodes where the flow of traffic is very high. Packet delays are for AODV \& DSR is increased when the mobility is reduced because of centrally located gateway nodes. Based on the traffic of number of relayed packet we have to decide which gateway can be used. This can be considered as a gateway selection problem. This causes the congestion at the media access control (MAC) level which in turn may lead to increase the high packet delays, since few nodes have to carry excessive loads. Such nodes may have higher battery power consumption which may cause degradation in routing performance. Along with that there are so many other problems on which this work will focus like: Gateway selection problem, Load balancing between the various gateways, Congestion Control. So this work will add an additional load effective routing mechanism IGTSM (Intermediate Gateway Traffic Sharing Model) which can consider and manage this congestion \& load control at the time of the route.
\end{abstract}

\section{General Terms}

MANET (Mobile Ad-Hoc Network); AODV (Ad-Hoc on Demand Distance Vector),

\section{Keywords}

IGTSM (Intermediate Gateway Traffic Sharing Model); WNM, Gateway, Load Distribution;

\section{INTRODUCTION}

Wireless mesh networks (WMNs) are very fast growing type of network technology for the last few years. In this the nodes consist of wireless mesh routers and mesh clients. Each node acts not only as an end user but also as a router, forwarding packets on behalf of other nodes whose destinations are not within its direct wireless transmission range [1]. Since Wireless Mesh Networks (WMNs) can be easily deployed without wire lines among wireless mesh routers, they allow us to quickly recover network access services in disaster areas even if the existing network infrastructures have been enormously destroyed by a terrible earthquake, tsunami, and so on. For this kind of network where disaster occurs the traffic model creation for optimizing routing overheads is a keen concern. Various studies have made these analysis traffic distributions approaches [2].
However, the performance of wireless mesh networks is largely affected by many factors, e.g., wireless mesh routers' locations, channel assignment, transmission scheduling, etc. In particular, the method of selecting gateways which has a connection to external networks significantly impacts on the network performance when the topology and routing have been fixed in the wireless mesh network. Congestion, load balancing, gateway selection, flow \& topology control is few of the problems which continually affect the network [3]. In this work, we suppose a wireless mesh network which consists of wireless mesh routers and a base station directly connected to external networks. Now between these heavy networks, the selection of heavy loaded nodes can be done by identifying it as a gateway. In this the base station is located at the center of the wireless mesh network chooses a certain number of wireless mesh routers as gateways, and establishes a connection with each of them. Our goal is to easily and quickly find the candidate gateways that maximize the system throughput without solving a complex optimization problem which includes a large number of parameters and involves heavy computation load. The performance of the proposed scheme is evaluated by numerical analysis, and demonstrated through computer simulations. In future the results show that our proposed scheme can determine the appropriate candidate gateway with high accuracy when there is a certain variation in the amount of traffic generated by users at each wireless mesh router.

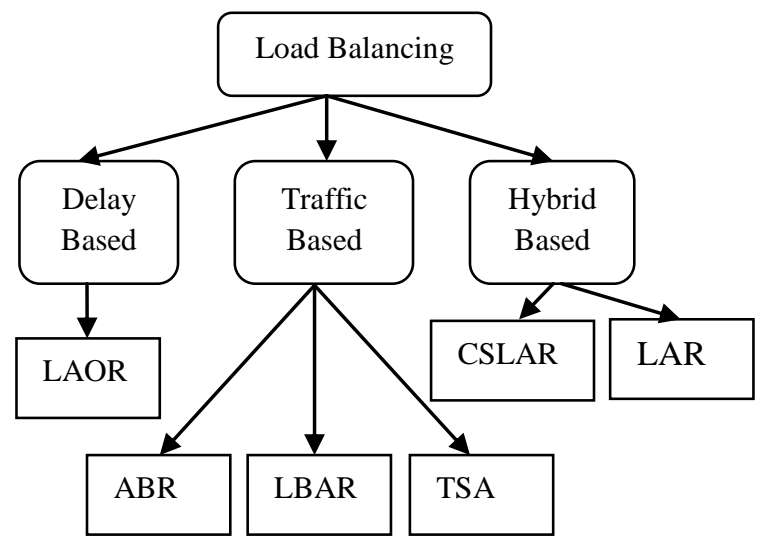

Figure 1: Load Balancing Routing Protocols

\section{BACKGROUND}

A load balancing protocol in MANET can be classified on the basis of the requirements of the network parameters \& variables which affects its utilization. These load balanced routing protocols for ad-hoc networks are based on various traffic scenarios which depend on demand and thus always 
combined with effective route discovery. In a broader context, the term load can be interpreted as:

(i) Channel load: Represents the load on the channel where multiple nodes contend to access the shared media.

(ii) Nodal load: Relates to a node's activity. Specifically, it refers to how busy a node is in processing, computation, and so on.

(iii) Neighboring load: Represents the load generated by communication activities among neighboring nodes.

The routing protocols can generally be categorized into three types (based on their load balancing techniques) as shown in Figure 1.

Delay-based: Where load balancing is achieved by attempting to avoid nodes with high link delay. An example is, LoadAware On-Demand Routing (LAOR).

Traffic-based: Where load balancing is achieved by evenly distributing traffic load among network nodes. Examples are Associatively Based Routing (ABR), Load Balanced Ad Hoc Routing (LBAR), and Traffic-Size Aware (TSA) scheme.

Hybrid-based: Where load balancing is achieved by combining the features of traffic- and delay-based techniques. Examples are Content Sensitive Load Aware Routing (CSLAR) and Load Aware Routing in Ad Hoc (LARA).

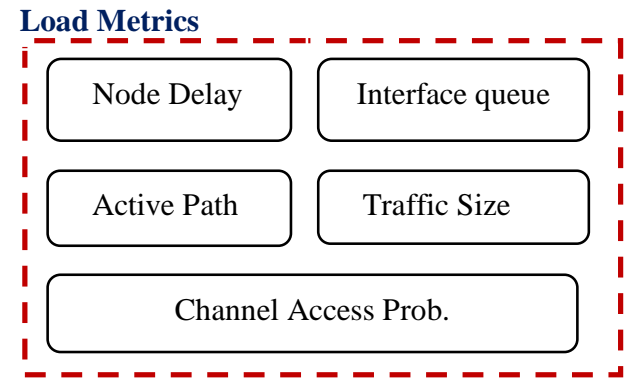

Figure 2: Load Metrics for distribution

\section{Load Balancing Required Metrics}

According to load balanced ad-hoc routing protocols are based on different load metrics shown in Figure 2.Given as:

(i) Active path: This refers to the number of active routing paths supported by a node. Generally, the higher the number of active routing paths, the busier the node since it is responsible for forwarding data packets from an upstream node to a downstream node.

(ii) Traffic size: This refers to the traffic load present at a node and its associated neighbors (measured in bytes).

(iii) Packets in interface queue: This refers to the total number of packets buffered at both the incoming and outgoing wireless interfaces.

(iv) Channel access probability: This refers to the likelihood of successful access to the wireless media. It is also related to the degree of channel contention with neighboring nodes.

(v) Node delay: This refers to the delays incurred in packet queuing, processing, and successful transmission.

\section{LITERATURE SURVEY}

This section describes the various approaches \& techniques which is used to solve various concerning problem of gateway based improvements in network performance. This study is categorized according to the basic requirement related to their specific areas. The extraction of the various approaches is:

Gateway Selection Problem
In [4] the author proposes a novel method to choose the gateway for deploying a WMN for disaster recovery, in achieving the maximum system throughput. The base station can select a number of wireless mesh routers as gateways, and establish a connection with each of them. Particularly, due to the base station supports one channel, this work assumes that mesh routers connect to each other by the single channel. Moreover, note that we consider only one gateway in a certain area. If there are multi-gateways, the problem can be solved by separating the nodes related to one gateway from nodes associated with other gateways The work suggested a novel minimum spanning tree based redundant path deletion which gives the exact path for routing.

The approach specifies the way to overcome gateway selection problem. Few other approaches have also suggested by taking different parameter for this selection problem like switching overhead with physical interference [5]. The scheme proposed is addressed in two parts, the first being the interference-aware link-channel scheduling algorithm which explicitly takes account of switching overhead and the second part put forth's a gateway selection algorithm. At initial level the scheme works effectively when compared to other existing gateway placement schemes like random, fixed and gridbased.

The gateway sharing can be balanced by effectively analyzing the optimal routing strategies which assumes that the traffic demands are static having exact prediction. The work gives a design, implement and evaluate two simple adaptive strategies to automatically select network paths and gateways for flows that dynamically arrive and depart, with the goal of achieving high network utilization and improved load balancing [6].

This work gives the design of the GARM routing metric that can effectively choose the best gateway for each mesh node [7]. This work considers a gateway-aware route in a WMN to consist of two parts: first, the multi-hop wireless path; second, the uplink at the gateway node. The work presents the metric design of these components, and then describes the unified metric. Given metrics are metric for the wireless path \& gateway capacity metric \& then later combining both to give GARM matrix from which we can be able to identify the best gateway. Some of the authors also work from giving the best decision in the case of multiple selection criteria of access points [8].

\section{Load Balancing Between Various Gateways}

This work will give a novel technique that balances the load among the different internet gateways in a WMN. The work uses three parameters to identify the best gateway between all the mesh nodes. The parameters are migration status, congestion notifies \& gateway table. It switches the point of attachment of an active source serviced gateway depending on the average queue length at the IGW. The proposed load balancing scheme includes: an initial gateway discovery module, which determines a primary gateway for a mesh router and a load balancing module that rebalances the load among the gateways. So the approach first does the selection \& then applies the balancing method to give better solutions [9].

This work [10] gives a distributed load balancing for gateway which exchanges information according to the sink requirement $\&$ the available demand of traffic. Gateways communicate with themselves through the wired network. Every time a demand change, the serving gateway notifies the rest of the gateways. Download traffic is routed based on this solution, i.e. a serving gateway is responsible for injecting the traffic of its sinks inside the WMN. To derive the demand of a 
sink, a gateway measures the throughput of flows destined to it. Since download traffic passes through the gateway, it can measure the throughput of the flows. To execute the loadbalancing algorithm, gateways must know the distance of every node to every gateway. Distance is measured as the length of the shortest path, with respect to a certain routing metric (e.g. Hop-count). Since in a WMN nodes are typically static (or quasi-static) this knowledge can be obtained easily by the gateways.

Gateway nodes act as a central point of connectivity to the wired infrastructure (typically the Internet). Therefore traffic aggregation occurs in the paths leading to a gateway and due to the limited wireless link capacity, these nodes are likely to be potential bottlenecks. This paper [11], propose a distributed load balancing algorithm to achieve load balancing on gateway nodes which leads to efficient traffic allocation as well as maximum use of network capacity. This algorithm uses Learning Automata in order to select the appropriate gateway node to send traffic. In general, the proposed algorithm (LALB) associates each router with a domain of its nearest gateways. Then each router that has data to send, select one gateway from its domain using its learning automaton. If the selected gateway node is appropriate, this act will be rewarded, otherwise, will be penalized which means to increase or reduce the probability of selecting that gateway respectively.

\section{Congestion Control}

While taking the various factors of the performance improvements of gateway congestion can be taken as a great factor to do that. It comes under the channel sharing of link assignment between multiple nodes. Since the number of channels is limited, some links in the WMNs may be allocated to the same channel. In this case, interference will occur if these links are close to each other. Interference between neighboring links can potentially cause network congestion. A multi-channel wireless mesh network with 4 channels uses TCP (Transmission Control Protocol), if the links become congested, there will be a reduction of the aggregated throughput. Thus, an efficient channel assignment algorithm is crucial to the reduction of interference due to neighboring transmissions using the same channel. This work formulates the Joint Optimal Channel Assignment and Congestion Control (JOCAC) as a decentralized utility maximization problem with constraints that arise from the interference of the neighboring transmissions. The work gives a formulation of the JOCAC problem by taking into account the available number of channels, the number of allocated NICs in each wireless router, the link's congestion price, transmission power, wireless path loss information, and channel frequency response [12].

Congestion control is unique because it has two prominent causes of failed transmissions which are difficult to tease apart lossy nature of the wireless medium and high extent of congestion around gateways in the network. The work addresses this issue to design an end-to-end congestion control mechanism for infrastructure wireless mesh networks. It formulates the congestion control problem and maps that to the restless multi-armed bandit problem, a well-known decision problem in the literature. To solve this, the work proposes three myopic policies to achieve a near-optimal solution to the mapped problem since no optimal solution is known for this problem. Therefore, it attempts to formulate the congestion control problem such that it can be mapped to a well-known decision problem called Multi-Armed Bandit (MAB) problem. The work analyses its solvability and propose some policies as there exists no solution in the literature for the mapped problem. We term the mapped problem as Multi-Armed Bandit Congestion Control (MABCC) problem [13].

Some of the authors had also given rule based congestion control mechanism like mesh adaptive pacing (MAP) [14]. It gives a proactive congestion control named as MAP, aims to avoid congestion before it actually occurs. The approach adapts TCP's transmission in order to overcome the spatial reuse constraint as well as the link layer unfairness of IEEE 802.11. Thereby, the transmission rate is adjusted proactively according to the current load in the network rather than reactively after a packet loss actually occurs. Since the proposed approach is only implemented at the wireless TCP sender and above the network layer of the mesh gateway, it is fully TCP-compatible. Specifically, the modifications of TCP in the wired domain or changes in IEEE 802.11 are not required. In general, the network entity in which adaptive pacing is implemented depends on the direction of a TCP flow. The work distinguishes between two flow directions: wireless-to-wired flows as well as wired-to-wireless flows \& gives the result according to the selected method.

In this paper [15] the author proposes a gateway rate control based mesh selective congestion control method which exploits the traffic patterns inherent in mesh networks. Since all traffic is expected to traverse the gateway, it enforces rate control there, anticipating that the sources will react to limit their traffic to their gateway-limited capacity. It will use a fair-share computer model to determine the appropriate rate for the various sources. The proposed approach does not require any additions or changes to the basic mesh network protocols, and works well with 802.11-based systems.

\section{PROBLEM STATEMENT}

Network topology can be restricted by the transmitting power at each node and the topology directly affects the load of the network. If the topology is too dense (i.e., nodes have more neighbors), there would be more choices for routing, but the power consumption of the system would be high. On the other hand, if the topology is too loose (i.e., with less edges), there would be less choices for routing (hence, some nodes could be overloaded) and the average hop-count between endnodes would be high. The goal is to find a balanced topology through Intermediate Gateway traffic sharing through proposed IGTSM that can meet end-users requirements and has minimum energy consumption.

The basic assumption is the involvement of nodes in data packet forwarding which depends on neighbors location based on inter \& intra route discovery mechanism. The existing approaches of node load balancing employs the fact that the broadcast for route request is not really a broadcast - it does not need to reach all nodes but only required a single destination. Therefore, the protocol drops route requests forwarding from the nodes in this heavy traffic routes.

The problems identified with the existing approaches are: Gateway selection problem

The problem comes under this module will be aimed to select the best gateway node among the others. It is based on the type of traffic \& its density.

(i) At the time of heavy traffic the distribution is unbalanced. Certain nodes have a greater frequency than other nodes.

(ii) In high connection scenarios shows the degradation in delivery ratio, relative overhead and end-to-end delay. 
Load Balancing between the various gateways

If the network has multiple gateways then there must be some sort of communication between them so as to synchronize the load sharing between them.

(iii) The gateway can mistakenly consider the temporary transmission problem as actual link breakage and the source is frequently notified of route breakages, which causes burden on network decrease performance \& throughput.

(iv) The load balancing is not independent, congestion occurs mainly at Intermediate Gateway.

\section{Congestion Control}

Due to congestion the performance of the gateway is affected. So to identify the best congestion \& flow control with an improved collision avoidance mechanism need to be proposed.

(v) Collisions and hidden node problem exist due to use of the unshared load medium.

(vi) Route stability is not achieved properly.

\section{PROPOSED IGTSM SOLUTION}

Considering the above mentioned problems this work proposes a novel design of a mechanism Intermediate Gateway Traffic Sharing Model (IGTSM) based load balancing for route discovery in MANET. The approach shows that the access delays cause the congestion at MAC layer \& computed with separate total delay using path selection metric. The proposed work can also be used for energy savings in routing with a threshold value which computed \& merge in the route request packet. The IGTSM is used as initial model of minimum load path selection criteria. Based on these parameters \& thresholds the algorithm balances load of the defined region and total path load. Effectively balancing the load of the particular path can be done by taking the number of packets delivered by any node as its load parameter. In IGTSM scheme Intermediate Gateways cannot send a route reply back to the source even if it has a route to the destination. Load of the neighboring nodes is known by the periodic broadcast of hello messages. the node i.e. number of packets delivered by it, and its flag bit starts with its time to leave (TTL) value

(ii) Each node at the time broadcasts hello message to its one hop neighboring nodes.

(iii) Give the assumption that multiple paths can be formed based on flooding.

(iv) The reply packet will consist of its node ID, the quantity of packets transmitted by it and its flag bit information with its TTL value

(v) The node receiving this hello message will record the values contained in the hello message, failing to receive hello message from earlier listed node denotes the node is no longer connected and the link is no longer valid and hence the its recorded value is deleted from the routing table

(vi) Classify the flow and identify the underutilized \& over utilized nodes.

(vii) Receiving hello message from the new node denotes a new link has been formed and its received values must be recorded in the routing table.

(viii) The destination node selects the best path among all the available paths through an effective route discovery mechanism

(ix) Network monitoring is performed in zero collision environments.

This work will develop separate algorithms for calculating the load at several nodes including all intermediate ones. These are Source Node Algorithm, Intermediate Gateway Algorithm and Destination Node Algorithm

\section{Benefits of Approach}

The given scheme can be able to:

a) Route the upload \& download traffic at Intermediate Gateway.

b) Network state monitoring can be performed based on congestion at the gateway or sink node.

c) The underutilized node problem can be solved.

d) Low complexity route balancing can be achieved.

e) Flow can be locked down or diverted at a specified time for a particular duration.

f) Flow classification can be done in a controlled manner
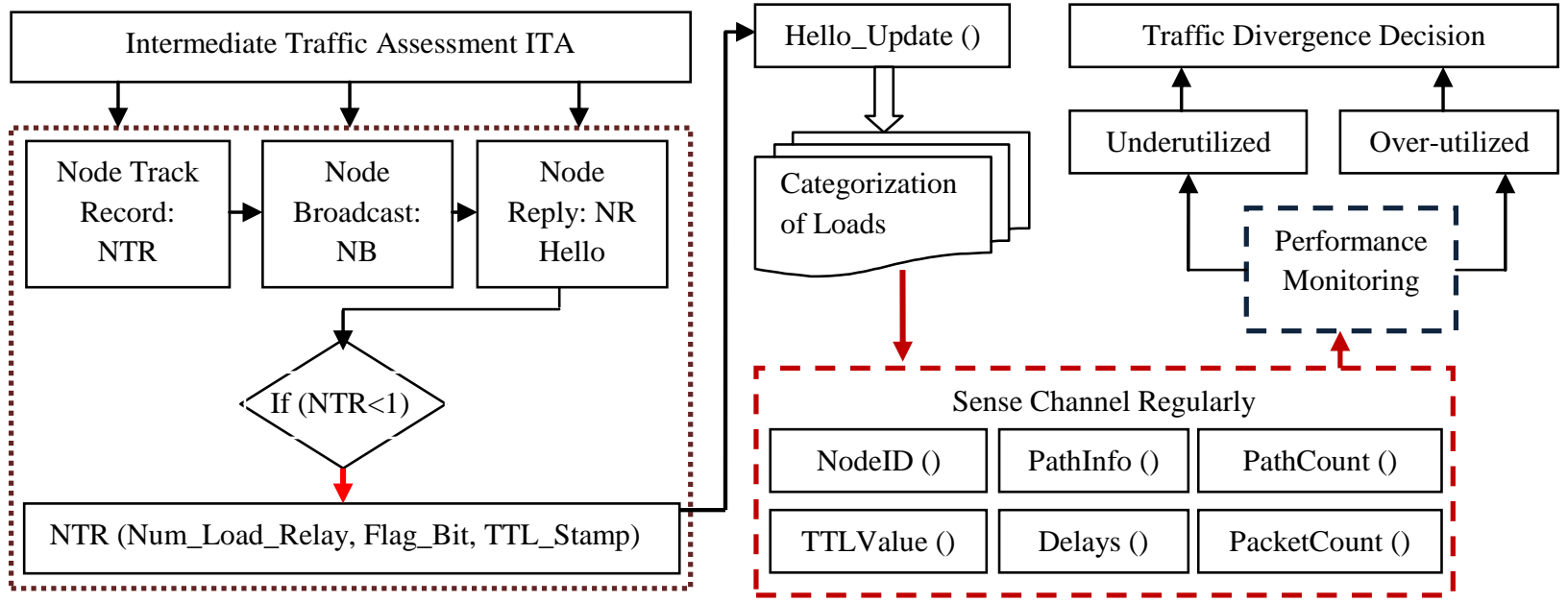

Figure 3: Proposed IGTSM Model Based Traffic Distribution

According to our proposed scheme IGTSM shown in Figure 3, the load balancing at various nodes can occur in following steps:

(i) Assume that each node keeps track of the information of its one hop neighbour such as amount of load relayed by

\section{EVALUATION PARAMETERS}

Experimental evaluation embodied work will shows the effectiveness of the proposed IGTSM algorithm. For network simulation, there are several performance metrics which are 
used to evaluate the performance. In future simulation purpose this work will use seven performance metrics for showing the expected results.

$>$ Packet Delivery Ratio

The packet delivery ratio is the ratio of the number of packets received at the destination to the number of packets sent from the source. The performance is better when packet delivery ratio is high.

$>$ Average end-to-end delay

This is the average time delay for data packets from the source node to the destination node. To find out the end-toend delay the difference of packets sent and received time was stored and then dividing the total time difference over the total number of packets received gave the average endto-end delay for the received packets. The performance is better when packet end to end delay is low.

$>$ Loss Packet Ratio (LPR)

Loss Packet Ratio is the ratio of the number of packets that never reached the destination of the number of packets originated from the source.

$>$ Efficiency: -The ratio of delivering data packets to those offered to the network.

$>$ Normalized Overhead Load:-The amount of control traffic generated (in bits) per data traffic delivered (in bits).

$>$ Average hop count: -Average number of hops travelled by data packets.

$>$ Average Discovery Period:-Average duration of a discovery period in seconds.

\section{CONCLUSION}

In the above proposed scheme of IGTSM based gateway load distribution model, the intermediate gateway can distribute their load to other gateways by path sharing based on their routing packet traffic. In this scheme each node checks its interface queue occupancy to determine whether it responds to the received RREQ or not. The criterion for the decision is a threshold value, which is calculated by each node when a RREQ is received. It shows the load analysis to each gateway. It is a variable along with the queue occupancy of the nodes around a backward path. The work decided a minimum threshold based limit up to which the gateways can transfer the packet \& after that it starts the IGTSM to share or distribute its load. Therefore, the threshold is adjusted adaptively according to the load status of the network this scheme can distribute the traffic evenly among the nodes in an ad hoc network.

Thus the main objective is to develop an enhancement for existing gateway selection \& congestion control to provide a contiguous balance of loading on the various types of node (Source, Intermediate, and Destination). At the initial level of research this work mainly focuses on three major domains; gateway selection problem, load balancing between the various gateways \& congestion control. Once the node traffic is decided for a gateway, distribution can be done in an intermediate specified amount of time, or congestion value. The congestion value is a user defined constraint placed on the algorithm and can be modified to meet the unique characteristics of the system, i.e., the battery life of individual nodes.

\section{FUTURE WORK}

Some problems and concepts that remain unaddressed can be performed in future. Such as with the help of pre-emptive approach more information can be added for exact timely analysis of load distributed. Unaddressed goals of the proposed algorithms that will achieve in the future are:
1. Optimal Path identification: -Minimize the number and size of the data structures required to implement the algorithms,

2. Flow control: -Extend the intermediate traffic calculation based on an input parameter,

3. Topology Control: -Allow every node equal opportunity to become an Intermediate Gateway in time,

4. Routing overhead reduction:-Maximize the stability in the network through minimizing the traffic

\section{ACKNOWLEDGMENT}

This work is proposed as the study level of PHD in wireless mesh network under the granted research at Pacific University. In this proposed IGTSM technique an effective gateway based load sharing method is given. It provides the various decision making factors which give the quality shifting of load through other available paths. The prominent factors of this work are gateway selection method, load sharing with optimal path \& improved congestion control. The proposed work is an initial level of research proposal which will give better results in future.

\section{REFERENCES}

[1] Juan J Galvez, Pedro M. Ruiz \& Antonio F.G. Skarmeta, "Responsive on-line gateway load balancing for wireless mesh network" in Elesevier Ad Hoc Network Journal, DOI: - 10.1016/j-adhoc.2012.06.002, 2012.

[2] Nils Aschenbruck, Matthias Frank, Peter Martini \& Jens T"olle, "Traffic Measurement and Statistical Analysis in a Disaster Area Scenario" in University of Bonn, 2005.

[3] Shitalkumar Jain \& Sunita I. Usturge, "Signal Strength Based Congestion Control in MANET “ in IISTE, ISSN 2225-0638, Vol 1, 2011.

[4] Wei Liu, Hiroki Nishiyama, Nei Kato, Yoshitaka Shimizu \& Tomoaki Kumagai, "A Novel Gateway Selection Method to Maximize the System Throughput of Wireless Mesh Network Deployed in Disaster Areas" in $23^{\text {rd }}$ IEEE International Symposium, 2012.

[5] Venkata Ganji, "Gateway Selection Scheme for Throughput Optimization in Multi-radio Multi-channel Wireless Mesh Networks Under Physical Interference Model" in Department of Computer Science Montana State Unviversity - Bozeman, 2012.

[6] Emilio Ancillotti, Raffaele Bruno \& Marco Conti, "Load-balanced Routing and Gateway Selection in Wireless Mesh Networks: Design, Implementation and Experimentation" in IEEE Transaction, ISSN 978-14244-7265-9/10, 2010.

[7] Prashanth A. K. Acharya, David L. Johnson \& Elizabeth M. Belding, "Gateway-aware Routing for Wireless Mesh Networks" in Dept of CSE, University of California.

[8] Shigeto Tajima, Teruo Higashino, Nobuo Funabiki \& Shoji Yoshida, "An Internet Gateway Access-Point Selection Problem forWireless Infrastructure Mesh Networks" in GSIST, Osaka University.

[9] Deepti Nandiraju, Lakshmi Santhanam, Nagesh Nandiraju, \& Dharma P. Agrawal, "Achieving Load Balancing in Wireless Mesh Networks Through Multiple Gateways" in IEEE Transaction 1-4244-0507-6/06, 2006. 
[10] Juan J Galvez, Pedro M. Ruiz \& Antonio F.G. Skarmeta, "A Distributed Algorithm for Gateway Load-Balancing in Wireless Mesh Networks" in IEEE Transaction, 2011.

[11] Maryam Kashanaki \& Zia Beheshti, "A Distributed Learning Automata based Gateway Load Balancing Algorithm in Wireless Mesh Networks" in Qazvin Azad University.

[12] A Hamed Mohsenian Rad and Vincent W.S. Wong, "Joint Optimal Channel Assignment and Congestion Control for Multi-channel Wireless Mesh Networks" in University of British Columbia.
[13] A B M. Alim Al Islam, S. M. Iftekharul Alam, Vijay Raghunathan, \& Saurabh Bagchi "Multi-Armed Bandit Congestion Control in Multi-Hop Infrastructure Wireless Mesh Networks" in Purdue University.

[14] Sherif M. ElRakabawy \& Christoph Lindemann, "Practical Rate-based Congestion Control for Wireless Mesh Networks" in University of Leipzig.

[15] Kamran Jamshaid, Lily Li, \& Paul A.S. Ward, "Gateway Rate Control of Wireless Mesh Networks" in ACM, ISSN: 159593472, 2006. 